\title{
Structural and Functional Connectivity of the Ascending Arousal Network for Prediction of Outcome in Patients with Acute Disorders of Consciousness
}

\section{Cesar O. Enciso-Olivera}

Fundación Universitaria de Ciencias de la Salud

Edgar G. Ordóñez-Rubiano

Fundación Universitaria de Ciencias de la Salud

Rosángela Casanova-Libreros

Fundación Universitaria de Ciencias de la Salud

Diana Rivera

Fundación Universitaria de Ciencias de la Salud

Carol J. Zarate-Ardila

Fundación Universitaria de Ciencias de la Salud Jorge Rudas

National University of Colombia

Cristian Pulido

National University of Colombia

Francisco Gómez

National University of Colombia

Darwin Martínez

Universidad Central

\section{Natalia Guerrero}

Fundación Universitaria de Ciencias de la Salud

Mayra A. Hurtado

Fundación Universitaria de Ciencias de la Salud Natalia Aguilera-Bustos

Fundación Universitaria de Ciencias de la Salud

Clara P. Hernández-Torres

Fundación Universitaria de Ciencias de la Salud José Hernandez

Fundación Universitaria de Ciencias de la Salud Jorge H. Marín-Muñoz ( $\nabla$ jmarin@imexhs.com ) Imaging Experts and Healthcare Services (ImexHS) 


\section{Research Article}

Keywords: diffusion tensor imaging (DTI), diagnosis, neurology

Posted Date: February 26th, 2021

DOl: https://doi.org/10.21203/rs.3.rs-244211/v1

License: (c) (1) This work is licensed under a Creative Commons Attribution 4.0 International License. Read Full License

Version of Record: A version of this preprint was published at Scientific Reports on November 25th, 2021. See the published version at https://doi.org/10.1038/s41598-021-98506-7. 


\section{Abstract}

Object: To determine the role of early acquisition of blood oxygen level-dependent (BOLD) signals and diffusion tensor imaging (DTI) for analysis of the connectivity of the ascending arousal network (AAN) in predicting neurological outcomes after acute traumatic brain injury (TBI), cardiopulmonary arrest (CPA), or stroke.

Methods: A prospective analysis of 50 comatose patients was performed during their ICU stay. Image processing was conducted to assess structural and functional connectivity of the AAN. Outcomes were evaluated after 3 and 6 months.

Results: Nineteen patients (38\%) had stroke, 18 (36\%) CPA, and $13(26 \%)$ TBI. Twenty-three patients were comatose $(44 \%), 11$ were in a minimally conscious state $(20 \%)$, and 16 had unresponsive wakefulness syndrome (32\%). Univariate analysis demonstrated that measurements of diffusivity, functional connectivity, and numbers of fibers in the gray matter, white matter, whole brain, midbrain reticular formation, and pontis oralis nucleus may serve as predictive biomarkers of outcome depending on the diagnosis. Multivariate analysis demonstrated a correlation of the predicted value and the real outcome for each separate diagnosis and for all the etiologies together.

Conclusion: Findings suggest that the above imaging biomarkers may have a predictive role for the outcome of comatose patients after acute TBI, CPA, or stroke.

\section{Introduction}

Predicting neurological outcomes and mortality in patients with acute disorders of consciousness (DOCs) is challenging ${ }^{1-3}$, and clinicians continue to question complex connection impairments of the ascending arousal network (AAN) in comatose patients ${ }^{2,4}$. Unfortunately, current clinical and radiological tools are not reliable for detecting consciousness or predicting recovery in those with either severe traumatic brain injury $(\mathrm{TBI})^{5}$, cardiac arrest ${ }^{3,6,7}$, or stroke ${ }^{7,8}$. To this end, multiple clinical and radiological tests have been proposed for assessing patients with DOCs, including bedside behavioral assessment ${ }^{9}$, electroencephalography $(\mathrm{EEG})^{10}$, task-based functional magnetic resonance imaging $(\mathrm{fMRI})^{11}$, restingstate-fMRI (rsfMRI) $)^{6,7,12}$, diffusion tensor imaging (DTI) ${ }^{1,2,7,8,13}$, and different combinations of these approaches $5,12,14$. In the absence of reliable prognostic tests, the clinician's judgment, experience, and communication skills may influence a family's decision about life-sustaining therapy and lead to premature care decisions before a patient's prognosis becomes clear ${ }^{14}$. In this regard, investigations have focused on diagnostic tests that might objectify this initial prediction assessment of consciousness outcome $e^{2,4,6,7}$.

The AAN is an essential component of human consciousness and is formed by a group of subcortical pathways connecting the rostral brainstem tegmentum to the hypothalamus, thalamus, and basal forebrain ${ }^{2,13}$. It has been described that DOCs after TBI, cardiac arrest, or stroke are related to axonal 
injury within the $\operatorname{AAN}^{1,13,15}$. Additionally, clinical and electrophysiological evaluations are insufficient and might be biased by sedation or any clinical condition, such as aphasia ${ }^{16}$. Accordingly, there is uncertainty concerning long-term effects in a broad spectrum of cognitive, behavioral, and functional impairments ${ }^{16}$. Overall, more specialized tests derived from MRI may be able to better characterize microstructural disturbances.

Blood oxygen level-dependent (BOLD) imaging utilizes a gradient-echo imaging sequence with parameters sensitive to the oxygen state of hemoglobin, which is used as contrast to delineate regional brain activity ${ }^{17}$. BOLD imaging allows for the analysis of both task-based fMRI and rsfMRI. rsfMRI itself can be used for studying different resting-state neural networks (RSNs) to establish functional connectivity in patients with a DOC ${ }^{12}$. On the other hand, DTI uses anisotropic diffusion to estimate the organization of brain tissue. Additionally, structural analysis of white matter (WM) with DTI techniques, including diffusion tensor tractography (DTT), has allowed physicians to scrutinize the anatomy of the $\mathrm{AAN}^{1,8,13,15,18}$, revealing the structural connectivity of this network ${ }^{1,19}$. Both tools can be employed to determine ascending and descending structural and functional connectivity between AAN brainstem nuclei and many different cortical areas ${ }^{12}$. Nevertheless, the exact biological nature of the structural and functional injury that leads to a DOC remains uncertain. Multiple efforts to elucidate the origin of impaired consciousness have led to the proposition of a compromised state for multiple cortical and subcortical areas and networks that may be involved in this process, including the brainstem ${ }^{20}$, thalamus $s^{6,21}$, hypothalamus ${ }^{1}$, frontal basal cortex ${ }^{1,4,5,13}$, and other association areas in the parietal lobe ${ }^{6}$. However, varying impairment in these areas may induce any DOC regardless of the etiology of the injury. In this regard, the aim of the present study was to analyze a combination of structural and functional information of the AAN obtained from both DTI and BOLD acquisitions to determine whether early acquisition of DTI and BOLD techniques for analysis of structural and functional connectivity of the AAN can predict neurological outcomes in terms of consciousness in patients with DOCs after TBI, cardiopulmonary arrest (CPA), or stroke.

\section{Results}

\section{Clinical Features}

Between October 2017 and January 2020, a total of 293 patients were assessed for eligibility criteria, of whom 50 were enrolled. Of the 243 excluded subjects, 104 were excluded for having a GCS score 8 or higher, 58 due to a previous history of any neurological or psychiatric disease, 41 because they were not able to be transferred to the MRI scanner due to their medical condition, 13 because MRI was not performed before death, 10 due to radiologically confirmed brain death during the first 48 hours after being admitted to the ICU, eight due to the family's decision not to participate, and nine due to other reasons (Fig. 1). The median age of the enrolled patients was 64 years (IQR: 49-74), and 27 (54\%) were female. Nineteen patients (38\%) were admitted with stroke, 18 (36\%) with hypoxic-ischemic brain injury after cardiac arrest, and 13 (26\%) with severe TBI. In regard to the state of consciousness, 23 patients 
were in coma (44\%), 11 in MCS (20\%), and 16 in UWS (32\%). The median length of stay in the ICU was 13.2 days (IQR: 5.1-21.3). The overall median ICU admission GCS score was 6 (IQR: $3-8$ ); the UWS group had a median score of 8 (IQR: 4-8), the coma group a median score of 6 (IQR: 3-7), and MCS a median score of 5 (IQR: $4-8$ ). The 28 patients (56\%) who were discharged from the ICU had a median GCS score of 9 (IQR: 3-12) (Table 1). Among them, 17 died during the follow-up before the neuropsychological evaluation in an outpatient setting. Additionally, 4 patients were lost from the study due to loss of contact with their surrogates. 
Table 1

Clinical and demographic features according to state of consciousness after ICU admission.

\begin{tabular}{|c|c|c|c|c|c|}
\hline & $\begin{array}{l}\text { Coma } \\
(n=23)\end{array}$ & $\begin{array}{l}\operatorname{MCS}(n= \\
11)\end{array}$ & $\begin{array}{l}\text { UWS }(n= \\
16)\end{array}$ & $\begin{array}{l}\text { Total } \\
(n=50)\end{array}$ & $\begin{array}{l}\mathrm{p} \text { - } \\
\text { value }\end{array}$ \\
\hline Age median (IQR) & $\begin{array}{l}50(72- \\
86)\end{array}$ & $\begin{array}{l}48(73- \\
90)\end{array}$ & $47(76-92)$ & $\begin{array}{l}49(74- \\
88)\end{array}$ & 0.42 \\
\hline Sex & & & & & 0.56 \\
\hline Female (\%) & $13(56.5)$ & $7(63.6)$ & $7(43.8)$ & $27(54)$ & \\
\hline Male (\%) & $10(43.5)$ & $4(36.4)$ & $9(56.2)$ & $23(46)$ & \\
\hline Education & & & & & 0.16 \\
\hline Illiterate (\%) & $0(0)$ & $0(0)$ & $1(6.3)$ & $0(0)$ & \\
\hline High School (\%) & $9(39.1)$ & $2(18.2)$ & $5(31.2)$ & $16(32)$ & \\
\hline Primary School (\%) & $10(43.5)$ & $5(45.5)$ & $2(12.5)$ & $17(34)$ & \\
\hline Technical (\%) & $0(0)$ & $2(18.2)$ & $2(12.5)$ & $4(8)$ & \\
\hline Graduate School (\%) & $4(17.4)$ & $2(18.2)$ & $6(37.5)$ & $12(24)$ & \\
\hline Comorbidities & & & & & 0.82 \\
\hline Hypertension (\%) & $7(30.4)$ & $7(63.6)$ & $8(50)$ & $22(44)$ & \\
\hline Diabetes Mellitus (\%) & $6(26.1)$ & $3(27.3)$ & $3(18.8)$ & $12(24)$ & \\
\hline Hypothyroidism (\%) & $2(8.7)$ & $2(18.2)$ & $3(18.8)$ & $7(14)$ & \\
\hline Dyslipidemia (\%) & $1(4.3)$ & $1(9.1)$ & $0(0)$ & $2(4)$ & \\
\hline Acute myocardial infarction (\%) & $0(0)$ & $1(9.1)$ & $1(6.3)$ & $2(4)$ & \\
\hline Other (\%) & $5(21.7)$ & $5(45.5)$ & $11(68.8)$ & $21(42)$ & \\
\hline $\begin{array}{l}\text { ICU admission GCS score median } \\
(\text { IQR) }\end{array}$ & $6(3-7)$ & $5(4-8)$ & $8(4-8)$ & $6(3-8)$ & 0.08 \\
\hline \multicolumn{6}{|l|}{ Intracranial Pressure } \\
\hline ICP monitoring (\%) & $2(8.7)$ & $1(9.1)$ & $1(6.25)$ & $4(8)$ & 0.95 \\
\hline Intracranial hypertension (\%) & $2(8.7)$ & $2(18.2)$ & $1(6.25)$ & $5(10)$ & 0.57 \\
\hline Mechanical ventilation (\%) & $22(95.7)$ & $10(90.9)$ & $14(87.5)$ & $46(92)$ & 0.65 \\
\hline Sepsis (\%) & $8(34.8)$ & $3(27.3)$ & $8(50)$ & $19(38)$ & 0.45 \\
\hline \multicolumn{6}{|c|}{ *Expenses not covered by their health insurance company in our institution } \\
\hline$P$ values correspond to comparat & sures a & the thr & ent groups. & & \\
\hline
\end{tabular}




\begin{tabular}{|c|c|c|c|c|c|}
\hline & $\begin{array}{l}\text { Coma } \\
(n=23)\end{array}$ & $\begin{array}{l}\operatorname{MCS}(n= \\
11)\end{array}$ & $\begin{array}{l}\text { UWS }(n= \\
16)\end{array}$ & $\begin{array}{l}\text { Total } \\
(\mathrm{n}=50)\end{array}$ & $\begin{array}{l}\mathrm{p}- \\
\text { value }\end{array}$ \\
\hline \multicolumn{6}{|l|}{ Vital status } \\
\hline Alive (\%) & $12(52.2)$ & $4(36.4)$ & $12(75)$ & $28(56)$ & 0.17 \\
\hline Deceased (\%) & $10(43.5)$ & $5(45.5)$ & $2(12.5)$ & $17(43)$ & \\
\hline $\begin{array}{l}{ }^{*} \text { Transferred to a different } \\
\text { institution (\%) }\end{array}$ & $0(0)$ & $1(9.1)$ & $2(12.5)$ & $3(6)$ & \\
\hline $\begin{array}{l}\text { GCS score at discharge median } \\
\text { (IQR) }\end{array}$ & $6(3-11)$ & $3(3-14)$ & $11(9-12)$ & $9(3-12)$ & 0.15 \\
\hline \multicolumn{6}{|c|}{ *Expenses not covered by their health insurance company in our institution } \\
\hline
\end{tabular}

\section{Structural and Functional Connectivity}

\section{Univariate Analysis}

A set of 60 individual imaging measurements was completed. However, only sixteen had an area under the curve (AUC) $\geq 0.80$. Thus, these specific features were explored as possible predictors with at least an accuracy of $80 \%$. A remarkable finding is that some single regions might serve as biomarkers of consciousness at ICU discharge. Figure 2 illustrates the potential use of single imaging measurements in specific regions to predict the patient's consciousness at ICU discharge. The ROC curve indicates possible use as an isolated approach for measuring specific regions to predict GCS score at ICU discharge.

Based on separate analysis for each variable, the gray matter (GM) FA was found to be a possible predictor in the setting of TBI. The AD, MD, and RD in both the GM and WM, as well as functional connectivity in the PON, and the number of fibers in the locus coeruleus (LC) and the parabrachial complex (PC) are possible predictors in the setting of CPA. Measurements of MD in the whole brain, WM, GM, MRF and PON, of AD in the WM, GM, and MRF, and the RD in the whole brain, WM, and MRF were found to be possible predictors of the state of consciousness at ICU discharge in the setting of a stroke. Separate analysis of automatic DTI, tractrography (Fig. 3) and BOLD measurements showed no predictive value for the state of consciousness at ICU discharge.

\section{Structural and Functional Connectivity - Multivariate Analysis}

Figure 4 illustrates the results obtained by GLM for each separate diagnosis; independent variables included the confounding variables and those features obtained from DTI and BOLD acquisitions. This figure shows the correlation between the real outcome and the value predicted by the model. In the setting of $\mathrm{TBI}$, the model reached an adjustment defined by the metric $\mathrm{R} 2=0.463$; it was 0.84 for stroke and 0.92 for CPA. Moreover, Fig. 5 denotes the correlation between the outcome and the model's predicted value 
from a global aspect, grouping patients with TBI, CPA, and stroke into one group. Three different scenarios were explored: (1) including both structural (DTI) and functional (BOLD) features, (2) functional features alone, and (3) structural features alone. The R2 values obtained for each scenario were 0.82 , 0.463 and 0.5 , respectively. These trends show the great importance of combining both features.

\section{Neurological Outcomes}

Thirty of the 50 patients died during follow-up before the neuropsychological evaluation. Of those remaining, five were lost during follow-up. Tests were performed for the remaining 15 patients. The NeuroPsi demonstrated that $9(60 \%)$ of the patients had normal psychological behavior, $1(6.6 \%)$ presented moderate psychological sequelae, and 5 (33.3\%) presented severe cognitive sequelae. Additionally, $40 \%$ presented abnormal orientation, $3(20 \%)$ had severe compromise of their attention/concentration, and $4(26.7 \%)$ and $5(33.3 \%)$ had moderate and severe visual memory impairment, respectively. On the other hand, the MoCa test showed that $11(73.3 \%)$ patients had cognitive deficits; 4 (26.7\%) had normal scores. Visual memory showed higher cognitive compromise, while the best performance was observed for language, attention, and orientation.

\section{Discussion}

\section{Conclusions}

Our findings suggest that early acquisition of BOLD and DTI for evaluation of structural and functional connectivity of the AAN may represent a tool for predicting outcome in patients with impaired consciousness after acute TBI, CPA, or stroke. DTI and BOLD analysis represent an observer- and operator-dependent task despite the automatic data processing involved, and clinical decision making must be made by physicians in a case-by-case manner.

\section{Limitations}

The limited number of patients recruited for this study represents a notable limitation. Although different etiologies were included, a separate analysis of each group was performed. This study sought to elucidate the prognostic value of early DTI and BOLD acquisitions, yet there are multifactorial limitations to enrolling comatose patients, including the high mortality in these scenarios as well as the social and economic background of a middle-income country. In addition, as this study lacks EEG data, further studies are needed to compare EEG findings with radiological biomarkers. Finally, specific analysis of the emotional, behavioral, and general neurological outcomes should also be addressed for targeted therapeutic assessment.

\section{Methods}

\section{Clinical Data and Study Design}


This is a prospective, observational, cohort-type diagnostic test study. Patients admitted to the ICU with acute DOC after CPA, stroke or TBI who stayed in the ICU for more than 48 hours were enrolled. Ten healthy volunteer adult subjects were recruited and analyzed as the control group ${ }^{12}$. Patients were admitted to the Hospital Infantil Universitario de San José ICU between October 2017 and January 2020. Inclusion criteria included patients over 18 years old, with either CPA treated within our institution with successful cardiopulmonary resuscitation, stroke (ischemic or hemorrhagic), or TBI, with a neurological evaluation prior to ICU admission consisting of coma (defined as Glasgow Coma Scale [GCS] score of $\leq$ $6 / 15$ without eye opening) after the initial resuscitation and who could be transferred to the MRI scanner. Exclusion criteria included patients diagnosed with brain death within the first 48 hours of admission to the ICU, those with severe TBI who were considered to be "nonsalvageable" by the neurosurgery staff, and those who had any medical history of a neurological entity prior to the event (e.g., trauma, degenerative disease), and those whose family decided to withdraw them from the study at any time during the followup period. Written informed consent for inclusion in the study was obtained from a surrogate for each patient. Authorization by our Institutional Ethics Board to include information for the subjects was requested. This research was performed in accordance with the Declaration of Helsinki. This prospective study was approved by our Institutional Review Board (Comité de Ética en Investigación con Seres Humanos - CEISH).

\section{Neurological Outcomes}

The initial bedside cognitive and behavioral assessment was performed by a neurologist (J.H.) in the first 24 hours or up to three days after the event whenever possible. The following tests were added for assessment by the patient's family: Lawton-Brody Instrumental Activities Scale, Frontal Systems Behavior Scale, and Memory Scale. Any sensory perception disorder was ruled out, and the family was asked about the patient's previous cognitive condition in relation to symptoms associated with previous behavioral changes, schooling, and occupation or any conditions that may bias the cognitive assessment. A subsequent evaluation was made by a neurologist (J.H.) and the ICU staff within the first 7 to 10 days after the initial injury or at the time of discharge from the ICU in the case of a short length of ICU stay. After the second assessment, the patients were categorized into those with coma, unresponsive wakefulness syndrome (UWS) (defined as a state of wakefulness without awareness in which there is preserved capacity for spontaneous or stimulus-induced arousal, evidenced by sleep-wake cycles and a range of reflexive and spontaneous behaviors, with complete absence of evidence for self or environmental awareness), or minimally conscious state (MCS) (defined as a state of severely altered consciousness in which minimal but clearly discernible behavioral evidence of self- or environmental awareness is demonstrated) ${ }^{45}$. Finally, the NeuroPSI (a short neuropsychological test battery for use with Spanish-speaking adults) ${ }^{46}$ and the Montreal Cognitive Assessment (MoCA) test ${ }^{47}$ were performed by a former neuropsychologist (C.P.H.) for cognitive function assessment at three- and six-month follow-ups whenever possible according to the patient's condition or a fatal outcome. Endpoints for evaluation were defined as an early consciousness status based on the average GCS score for the previous two days before ICU discharge, middle-term consciousness status based on the ability or not to perform the MoCA test (awareness of their selves or their environment) during follow-up, or a fatal outcome. 


\section{Neuroimaging Data Acquisition}

A 1.5-T General Electric scanner was used for data acquisition. As reported previously ${ }^{12}$, we acquired one hundred and eighty multislice T2*-weighted functional images using an axial slice orientation and covering the whole brain (slice thickness $=4.5 \mathrm{~mm}$ without free space, matrix $=64 \times 64 \mathrm{~mm}, \mathrm{TR}=3000$ $\mathrm{ms}, \mathrm{TE}=60 \mathrm{~ms}$, flip angle $=90^{\circ}$ and FOV $=288 \times 288 \mathrm{~mm}$ ). The three initial volumes were discarded to avoid T1 saturation effects. Moreover, axial diffusion weighted imaging (DWI) (slice thickness $=2.5 \mathrm{~mm}$ without free space, matrix $=100 \times 100, \mathrm{TR}=17000 \mathrm{~ms}, \mathrm{TE}=96 \mathrm{~ms}$, flip angle $=90^{\circ}, \mathrm{FOV}=250 \times 250 \mathrm{~mm}$, $\mathrm{b}$ value $=1000$ and gradient directions $=30$ ) was acquired. Finally, structural axial T1 (slice thickness $=1$ $\mathrm{mm}, \mathrm{GAP}=1 \mathrm{~mm}$, matrix $=256 \times 256 \mathrm{~mm}, \mathrm{TR}=670 \mathrm{~ms}, \mathrm{TE}=22 \mathrm{~ms}$, flip angle $=20^{\circ}$ and FOV $=250 \times 250$ $\mathrm{mm}$ ) and axial T2 (slice thickness $=6 \mathrm{~mm}, \mathrm{GAP}=1 \mathrm{~mm}$, matrix $=320 \times 320 \mathrm{~mm}, \mathrm{TR}=6.000 \mathrm{~ms}, \mathrm{TE}=96$ $\mathrm{ms}$, flip angle $=90^{\circ}$ and FOV $=220 \times 220 \mathrm{~mm}$ ) images were acquired for anatomical reference.

\section{Neuroimaging Data Preprocessing}

The T1 and rsfMRI data were preprocessed using the approach suggested by Kandeepan et al. ${ }^{48}$ In particular, T1 preprocessing included manual removal of the neck, brain extraction using FSL ${ }^{49}$, correction of low-frequency intensity nonuniformity based on the $\mathrm{N} 4$ bias field correction algorithm from SimpleITK ${ }^{50}$, image denoising based on the nonlocal means algorithm from Dipy ${ }^{50,51}$, and spatial normalization to standard stereotactic Montreal Neurological Institute (MNI) space using the SPM12 normalization algorithm ${ }^{50-52}$. The initial six volumes of the fMRI data were discarded to avoid T1 saturation effects. Head motion and slice timing corrections were performed on the fMRI data using FSL, followed by artifact correction using RapidArt ${ }^{53}$. Subsequently, the fMRI data were coregistered to a T1 image using SPM12 and spatially normalized to the MNI space using the SPM12 normalization algorithm. Finally, spatial smoothing of the fMRI data was performed with a Gaussian kernel of $8 \mathrm{~mm}$ full width at half maximum, as implemented in SPM12. The spurious variance was reduced by regression of nuisance waveforms derived from time series extracted from regions of noninterest (WM and cerebrospinal fluid). Additional nuisance regressors included the blood oxygen level-dependent imaging (BOLD) time series averaged over the whole brain. The DWI images were preprocessed using the approach suggested by Parra-Morales et $a{ }^{12}$. This process included automatic realignment, correction of eddy-current artifacts by using FSL tools, reslicing to obtain the isotropic voxel size, automatic brain extraction by the BET tool from FSL, and improvement of signal-to-noise rate using the Non-Local mean algorithm from Dipy.

\section{Location and Characterization of Regions of Interest}

Regions of interest (ROIs) for AAN reconstruction were located based on Harvard Ascending Arousal Network Atlas provided by the Martinos Center for Biomedical Imaging, Charleston, Massachusetts, USA $^{13}$, and the cortex nuclei were extracted from Harvard-Oxford Atlas provided by the Harvard Center for Morphometric Analysis ${ }^{54}$. These nuclei were linearly (rigid, translation, and affine transformation) and nonlinearly (symmetric diffeomorphic registration) ${ }^{55}$ registered with each subject space. 


\section{AAN Functional Connectivity}

Many features were extracted from the distinct imaging acquisition techniques to characterize the connectivity of the AAN with the rest of the brain. Functional connectivity (FC) was estimated using a measure of Pearson's correlation among the average filtered time courses of eight AAN nuclei and fortyeight cortical nuclei ${ }^{54}$. A bandpass Butterworth filter with cutoff frequencies set at $0.005 \mathrm{~Hz}$ and $0.1 \mathrm{~Hz}$

was used for this step ${ }^{56}$, which produced three hundred eighty-four FC values (8 AAN nuclei $x 48$ cortical nuclei). These sets of values were summarized by averaging the quantities to eight representative values associated with each AAN nucleus.

\section{AAN Structural Connectivity}

A constant solid angle model was used to obtain directions from diffusion imaging ${ }^{57}$. This model estimates the orientation distribution function (ODF) at each voxel. This ODF is a function of the distribution of water movement, and its peaks are a suitable estimate for the orientation of each tract at each voxel. Afterwards, a deterministic local fiber tracking algorithm was applied for the $\mathrm{WM}^{58}$, with the following set of parameters: min separation angle $=30^{\circ}$, step size $=1$ and number of seeds in each voxel $=8$. Subsequently, the number of tracts that may connect every possible pair of nuclei between the AAN and the cortex was calculated (8 AAN nuclei x 48 cortical nuclei). These sets of values were also averaged to obtain the eight representative quantities associated with each AAN nucleus.

\section{Statistical Analysis}

\section{Univariate Analysis}

To assess the predictive ability of image-based features, univariate analysis of each single value from the set of 60 measurements was performed through a receiver operating characteristic (ROC) curve (Fig. 2). The measurements were grouped according to the diagnosis, and the outcome was established as indicated in the previous section. The associated area under the curve was then used to determine the predictive ability of a single image-based feature linked to the admission diagnosis.

\section{Multivariate Analysis}

A general linear model (GLM) was employed for multivariate analysis. GLM is able to quantify the variation of a dependent variable in terms of a linear combination of several reference independent variables $^{59}$. GLM was implemented as follows: 
$Y_{\text {outcome }}=\beta^{f M R I} X^{f M R I}+\beta^{D T I} X^{D T I}+\beta^{\text {confoundings }} X^{\text {confoundings }}+e$

where $Y_{\text {outcome }}$ is a column vector that contains the mean GCS score for the last two days before ICU discharge; $X^{f M R I}, X^{D T I}$ and $X^{\text {confoundings }}$ are matrices that contain for each column the quantitative value for each feature from fMRI, DTI and confounding data, respectively; $e$ represents the error during model fit; $\beta^{f M R I}, \beta^{D T I}$ and $\beta^{\text {confoundings }}$ represent the weight in the registration model for each $\mathrm{fMRI}, \mathrm{DTI}$ and confounding variable. The $\mathrm{R}^{2}$ value was used to determine the level of model fit for each case. Finally, p-values were used to determine the significance level for each variable explaining dependent variables.

\section{Declarations}

\section{Data Availability}

The data that support the findings of this study are available on request from the corresponding author, [JHM]. The data are not publicly available due to the containing information that could compromise the privacy of research participants (e.g. Patients' names, surrogates' names, IDs).

\section{Competing Interests}

This article is funded by the Administrative Department of Science, Technology, and Innovation of the government of Colombia (Colciencias) under grant number 702-2016.

\section{Author Contributions}

Conceptualization: C.E.O., J.H.M, F.G, E.G.O. Data acquisition: C.E.O., C.J.Z., M.A.H., N.G., C.P., N.A., C.P.H., J.H., Formal analysis: R.C.L, D.R., J.R., C.P., F.G., D.M, C.P.H. E.G.O., J.H.M. Statistical analysis: R.C.L., D.R., J.R., D.M. Writing (original draft): C.E.O, E.G.O., C.J.Z., J.R., D.M., F.G, J.M. Writing (critical review): all authors. All authors read and approved the final manuscript.

\section{Acknowledgements}

To all health personnel who significantly contributed to the acquisition and processing of the data used for this study, including nurses, anesthesiologists, psychologists, and technicians. To all patients and surrogates who contribute to the enrollment process for this study.

\section{References}


1. Ordonez-Rubiano, E. G. et al. Reconstruction of the ascending reticular activating system with diffusion tensor tractography in patients with a disorder of consciousness after traumatic brain injury. Cureus. 9, e1723 (2017).

2. Snider, S. B. et al. Disruption of the ascending arousal network in acute traumatic disorders of consciousness. Neurology. 93, e1281-e1287 (2019).

3. Koenig, M. A. et al. MRI default mode network connectivity is associated with functional outcome after cardiopulmonary arrest. Neurocrit. Care. 20, 348-357 (2014).

4. Edlow, B. L. et al. Personalized connectome mapping to guide targeted therapy and promote recovery of consciousness in the intensive care unit. Neurocrit. Care. 33, 364-375 (2020).

5. Edlow, B. L. et al. Early detection of consciousness in patients with acute severe traumatic brain injury. Brain. 140, 2399-2414 (2017).

6. Wagner, F. et al. Outcome prediction with resting-state functional connectivity after cardiac arrest. Sci. Rep. 10, 11695 (2020).

7. Velly, L. et al. Use of brain diffusion tensor imaging for the prediction of long-term neurological outcomes in patients after cardiac arrest: a multicentre, international, prospective, observational, cohort study. Lancet Neurol. 17, 317-326 (2018).

8. Jang, S. H., Chang, C. H., Jung, Y. J., Kim, J. H. \& Kwon, Y. H. Relationship between impaired consciousness and injury of ascending reticular activating system in patients with intracerebral hemorrhage. Stroke. 50, 2234-2237 (2019).

9. Monti, M. M. et al. Willful modulation of brain activity in disorders of consciousness. N. Engl. J. Med. 362, 579-589 (2010).

10. Kondziella, D. et al. European academy of neurology guideline on the diagnosis of coma and other disorders of consciousness. Eur. J. Neurol. 27, 741-756 (2020).

11. Owen, A. M. et al. Detecting awareness in the vegetative state. Science. 313, 1402 (2006).

12. Parra-Morales, A. M. et al. Structural and functional connectivity of ascending reticular activating system in a patient with impaired consciousness after a cardiac arrest: a case report. Med. (Baltim). 98, e15620 (2019).

13. Edlow, B. L. et al. Neuroanatomic connectivity of the human ascending arousal system critical to consciousness and its disorders. J. Neuropathol. Exp. Neurol. 71, 531-546 (2012).

14. Edlow, B. L. \& Fins, J. J. Assessment of covert consciousness in the intensive care unit: clinical and ethical considerations. J. Head Trauma Rehabil. 33, 424-434 (2018).

15. Jang, S. H., Kim, O. L., Kim, S. H. \& Kim, J. B. The relation between loss of consciousness, severity of traumatic brain injury, and injury of ascending reticular activating system in patients with traumatic brain injury. Am. J. Phys. Med. Rehabil. 98, 1067-1071 (2019).

16. Galanaud, D. et al. Assessment of white matter injury and outcome in severe brain trauma: a prospective multicenter cohort. Anesthesiology. 117, 1300-1310 (2012). 
17. Glover, G. H. Overview of functional magnetic resonance imaging. Neurosurg. Clin. N. Am. 22, 133139, vii(2011).

18. Jang, S. H. \& Kwon, H. G. Injury of ascending reticular activating system associated with delayed post-hypoxic leukoencephalopathy: a case report. BMC Neurol. 17, 139 (2017).

19. Tan, X. et al. Structural connectome alterations in patients with disorders of consciousness revealed by 7-tesla magnetic resonance imaging. Neuroimage Clin. 22, 101702 (2019).

20. Ordonez-Rubiano, E. G. et al. Recovery of consciousness after a brainstem cavernous malformation hemorrhage: a descriptive study of preserved reticular activating system with tractography. J. Clin. Neurosci. 59, 372-377 (2019).

21. Hindman, J. et al. Thalamic strokes that severely impair arousal extend into the brainstem. Ann. Neurol. 84, 926-930 (2018).

22. Pujol, J. et al. Largest-scale dissociation of brain activity at propofol-induced loss of consciousness. Sleep. https://doi.org/10.1093/sleep/zsaa152 (2020).

23. Nir, T. et al. Resting-state functional connectivity in early postanaesthesia recovery is characterised by globally reduced anticorrelations. Br. J. Anaesth. 125, 529-538 (2020).

24. Alexander, A. L., Lee, J. E., Lazar, M. \& Field, A. S. Diffusion tensor imaging of the brain. Neurotherapeutics. 4, 316-329 (2007).

25. Xiong, K. L., Zhu, Y. S. \& Zhang, W. G. Diffusion tensor imaging and magnetic resonance spectroscopy in traumatic brain injury: a review of recent literature. Brain Imaging Behav. 8, 487-496 (2014).

26. Basser, P. J. \& Jones, D. K. Diffusion-tensor MRI: theory, experimental design and data analysis - a technical review. NMR Biomed. 15, 456-467 (2002).

27. Hulkower, M. B., Poliak, D. B., Rosenbaum, S. B., Zimmerman, M. E. \& Lipton, M. L. A decade of DTI in traumatic brain injury: 10 years and 100 articles later. AJNR Am. J. Neuroradiol. 34, 2064-2074 (2013).

28. Zhang, J. et al. Correlations between diffusion tensor imaging and levels of consciousness in patients with traumatic brain injury: a systematic review and meta-analysis. Sci. Rep. 7, 2793 (2017).

29. Hammoud, D. A. \& Wasserman, B. A. Diffuse axonal injuries: pathophysiology and imaging. Neuroimaging Clin. N. Am. 12, 205-216 (2002).

30. de la Marquez, C. D. et al. Diffusion tensor imaging biomarkers for traumatic axonal injury: analysis of three analytic methods. J. Int. Neuropsychol. Soc. 17, 24-35 (2011).

31. Benson, R. R. et al. Global white matter analysis of diffusion tensor images is predictive of injury severity in traumatic brain injury. J. Neurotrauma. 24, 446-459 (2007).

32. Tang, C. Y. et al. Diffuse disconnectivity in tBi: a resting state fMri anD Dti stuDy. Transl. Neurosci. 3, 9-14 (2012).

33. Sekhon, M. S., Ainslie, P. N. \& Griesdale, D. E. Clinical pathophysiology of hypoxic ischemic brain injury after cardiac arrest: a "two-hit" model. Crit. Care. 21, 90 (2017). 
34. Thornton, J. S. et al. Anisotropic water diffusion in white and gray matter of the neonatal piglet brain before and after transient hypoxia-ischaemia. Magn. Reson. Imaging. 15, 433-440 (1997).

35. Samuels, E. R. \& Szabadi, E. Functional neuroanatomy of the noradrenergic locus coeruleus: its roles in the regulation of arousal and autonomic function part I: principles of functional organisation. Curr. Neuropharmacol. 6, 235-253 (2008).

36. Chiang, M. C. et al. Parabrachial complex: a hub for pain and aversion. J. Neurosci. $\mathbf{3 9}, \mathbf{8 2 2 5 - 8 2 3 0}$ (2019).

37. Klatzo, I. Pathophysiological aspects of brain edema. Acta Neuropathol. 72, 236-239 (1987).

38. Sotak, C. H. The role of diffusion tensor imaging in the evaluation of ischemic brain injury - a review. NMR Biomed. 15, 561-569 (2002).

39. Chaudhary, N. et al. Diffusion tensor imaging in hemorrhagic stroke. Exp. Neurol. 272, 88-96 (2015).

40. Davis, D. et al. Rapid monitoring of changes in water diffusion coefficients during reversible ischemia in cat and rat brain. Magn. Reson. Med. 31, 454-460 (1994).

41. Mintorovitch, J. et al. Comparison of diffusion- and T2-weighted MRI for the early detection of cerebral ischemia and reperfusion in rats. Magn. Reson. Med. 18, 39-50 (1991).

42. Fridman, E. A., Beattie, B. J., Broft, A., Laureys, S. \& Schiff, N. D. Regional cerebral metabolic patterns demonstrate the role of anterior forebrain mesocircuit dysfunction in the severely injured brain. Proc. Natl. Acad. Sci. U. S. A. 111, 6473-6478(2014).

43. Threlkeld, Z. D. et al. Functional networks reemerge during recovery of consciousness after acute severe traumatic brain injury. Cortex. 106, 299-308 (2018).

44. Edlow, B. L., Claassen, J., Schiff, N. D. \& Greer, D. M. Recovery from disorders of consciousness: mechanisms, prognosis and emerging therapies. Nat. Rev. Neurol. https://doi.org/10.1038/s41582020-00428-x (2020).

45. Turner-Stokes, L. Prolonged disorders of consciousness: new national clinical guidelines from the Royal College of Physicians, London. Clin. Med. (Lond.). 14, 4-5 (2014).

46. Ostrosky-Solis, F., Ardila, A. \& Rosselli, M. NEUROPSI: a brief neuropsychological test battery in Spanish with norms by age and educational level. J. Int. Neuropsychol. Soc. 5, 413-433 (1999).

47. Nasreddine, Z. S. et al. The montreal cognitive assessment, MoCA: a brief screening tool for mild cognitive impairment. J. Am. Geriatr. Soc. 53, 695-699 (2005).

48. Kandeepan, S. et al. Modeling an auditory stimulated brain under altered states of consciousness using the generalized Ising model. Neuroimage. 223, 117367 (2020).

49. Woolrich, M. W. et al. Bayesian analysis of neuroimaging data in FSL. Neuroimage. 45 (1 Suppl), S173-186 (2009).

50. Beare, R., Lowekamp, B. \& Yaniv, Z. Image segmentation, registration and characterization in R with SimpleITK. J. Stat. Softw. 86, 8 (2018).

51. Garyfallidis, E. et al. Dipy, a library for the analysis of diffusion MRI data. Front. Neuroinform. 8, 8 (2014). 
52. Penny, W., Friston, K., Ashburner, J., Hiebel, S. \& Nichols, T. Statistical parametric mapping: the analysis of functional brain images(Elsevier, 2011).

53. NITRC. NITRC: Neurolmaging Tools \& Resources Collaboratory https://www.nitrc.org/plugins/mwiki/index.php/nitrc:Site_Map (2020).

54. Desikan, R. S. et al. An automated labeling system for subdividing the human cerebral cortex on MRI scans into gyral based regions of interest. Neuroimage. 31, 968-980 (2006).

55. Avants, B. B., Epstein, C. L., Grossman, M. \& Gee, J. C. Symmetric diffeomorphic image registration with cross-correlation: evaluating automated labeling of elderly and neurodegenerative brain. Med. Image Anal. 12, 26-41 (2008).

56. Demertzi, A. et al. Multiple fMRI system-level baseline connectivity is disrupted in patients with consciousness alterations. Cortex. 52, 35-46 (2014).

57. Aganj, I. et al. Reconstruction of the orientation distribution function in single- and multiple-shell qball imaging within constant solid angle. Magn. Reson. Med. 64, 554-566 (2010).

58. Afzali, M., Fatemizadeh, E. \& Soltanian-Zadeh, H. Interpolation of orientation distribution functions in diffusion weighted imaging using multi-tensor model. J. Neurosci. Methods. 253, 28-37 (2015).

59. Monti, M. M. Statistical analysis of fMRI time-series: a critical review of the GLM approach. Front. Hum. Neurosci. 5, 28 (2011).

\section{Figures}




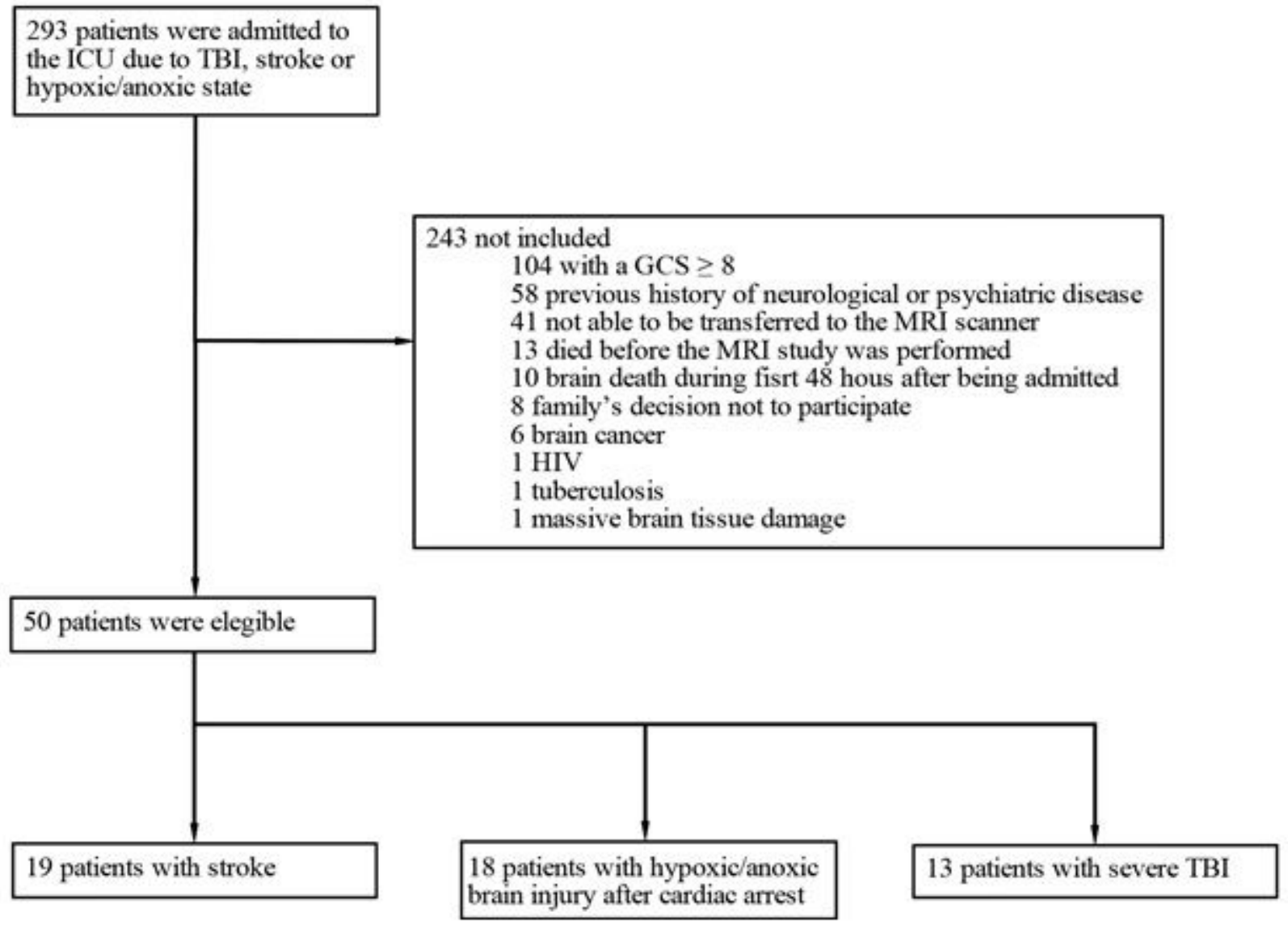

\section{Figure 1}

Description of the enrollment of patients in the study.
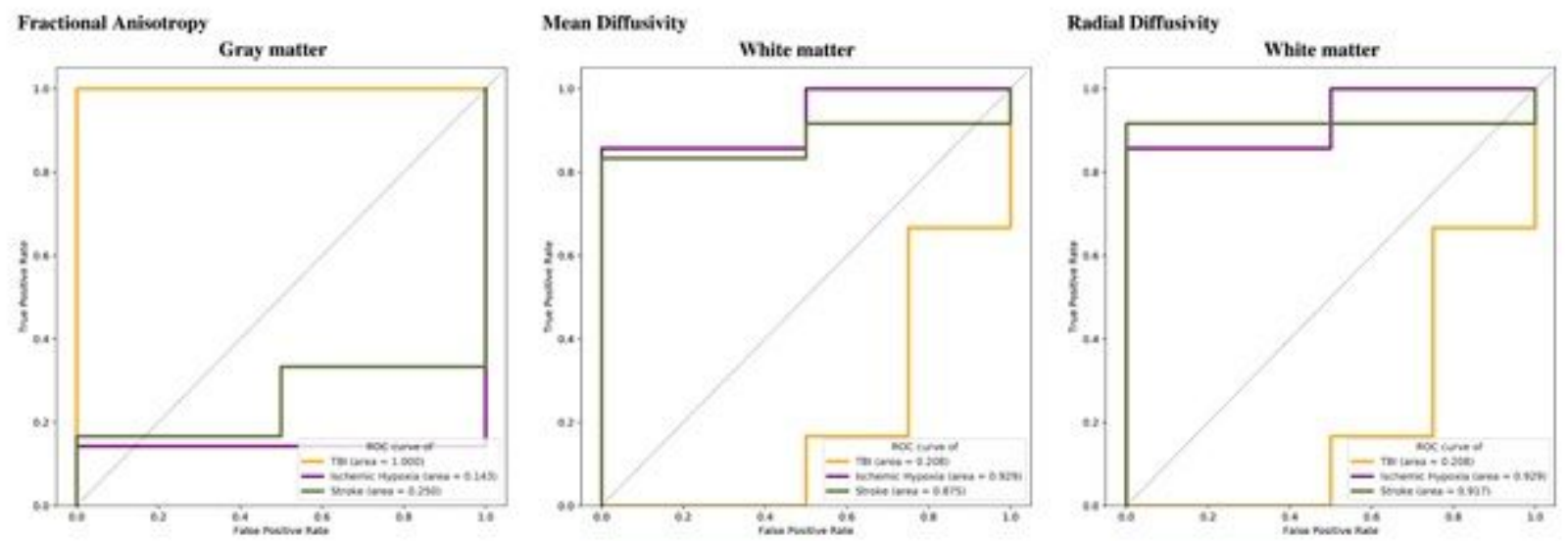

Figure 2

Receiver operating characteristic (ROC) curves of single measurements for different cortical and subcortical areas. ROC curves demonstrate the possible predictive value of data comparing true positive 
and false positive rates for fractional anisotropy, mean diffusivity, and radial diffusivity among patients with TBI (yellow), cardiac arrest (purple), and stroke (green).

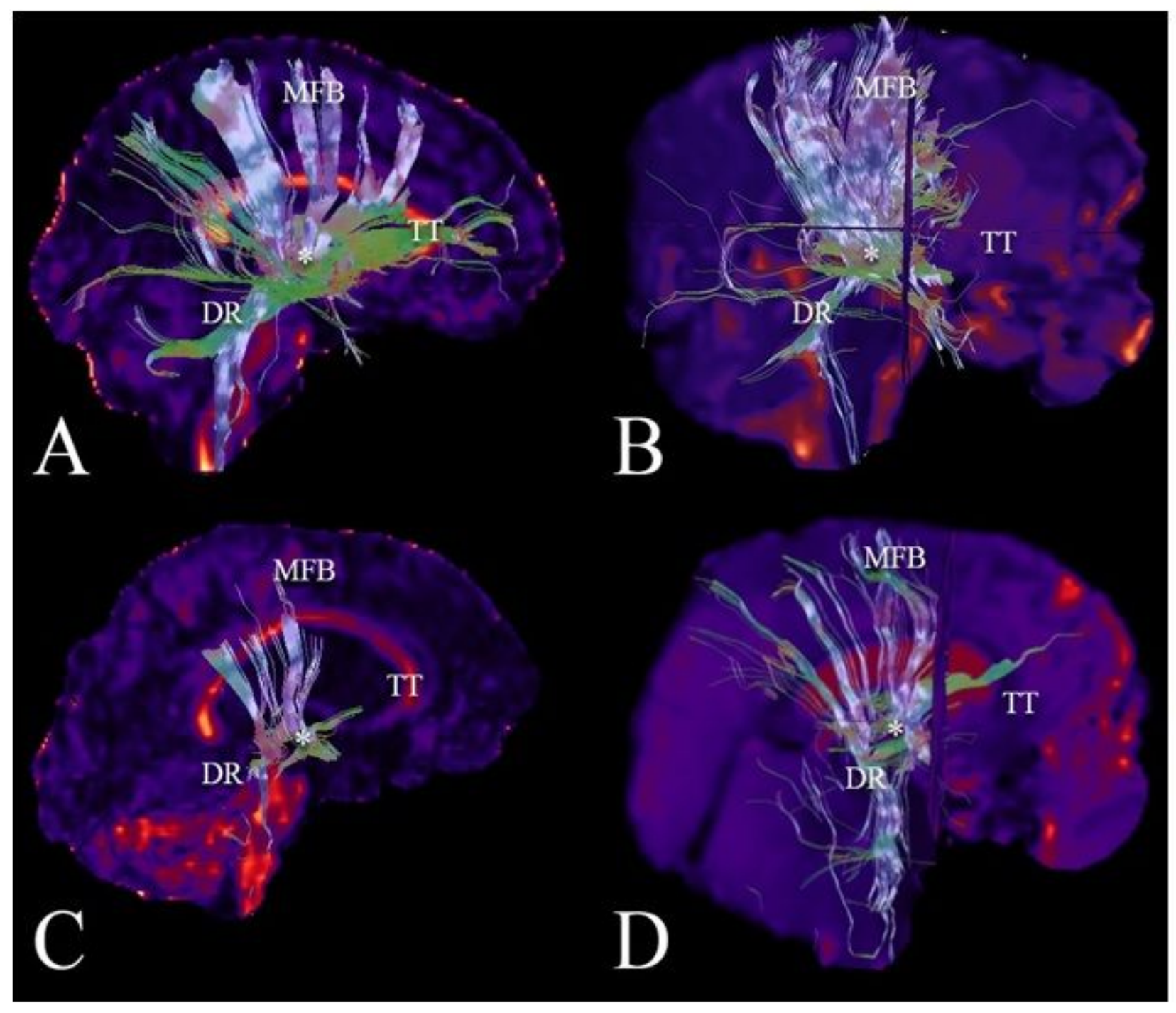

\section{Figure 3}

Reconstruction of the tractography of ascending arousal network. (A) A complete reconstruction of the AAN of a normal subject is demonstrated. Major components including the medial forebrain bundle (MFB) projecting to the dorsal frontal cortex, the dorsal raphe (DR), the thalamic-hypothalamic complex (asterisk), and superior and inferior tegmental tracts (TT) projecting to the basal frontal cortex are shown. (B) A reconstruction of the AAN of a comatose patient after a severe traumatic brain injury is observed, denoting a destruction of the tegmental tracts. (C) A reconstruction of the AAN of a patient after a stroke demonstrates a disruption of the tracts in the length of the MFB, the fibers of the DR, and the tegmental tracts. (D) A reconstruction of the AAN is shown in a comatose patient after a cardiac arrest, demonstrating a decrease in the number of fibers of all components of the AAN. 
TBI

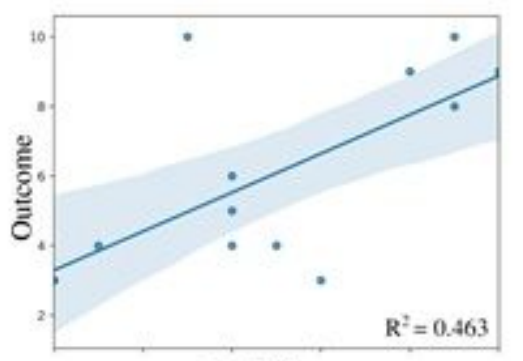

Prediction
Hypoxic-ischemic

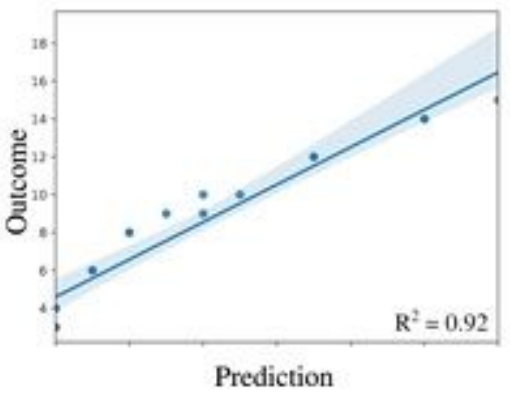

Stroke

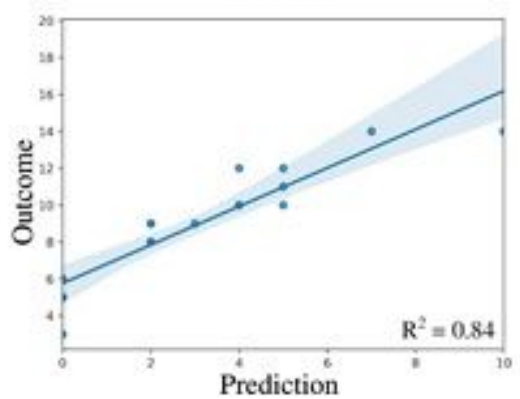

Figure 4

Multivariate analysis for prediction of outcomes of consciousness by etiology. In the three scenarios (traumatic brain injury, cardiac arrest, and stroke), the outcome for each patient is demonstrated by a linear model. The curves show a direct correlation between imaging and prediction of consciousness outcome, with corresponding R2 values of $0.463,0.92$ and 0,84 , respectively.
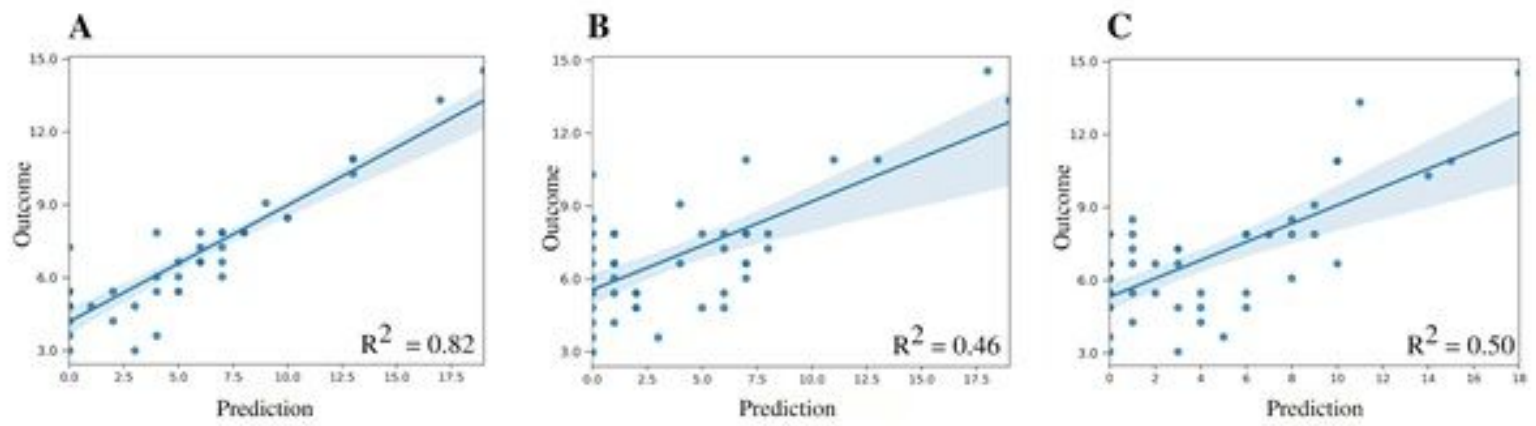

Figure 5

Multivariate prediction at the group level. The relationship between outcome and prediction of the general linear model is demonstrated using (a) both the structural and functional descriptors together, (b) the functional descriptors alone, and (c) the structural descriptors alone. The corresponding R2 values are $0.82,0.46$ and 0.5 , respectively. 